\title{
Distribution Network Data-Monitoring System based on Wireless Sensor Networks
}

\author{
Shihai Ma ${ }^{1}$, Yan Zhao ${ }^{1}$ and Haiyan Zhang $^{1}$ \\ 1 Shenyang Institute of Engineering, Shenyang 110136, China \\ Email:mash@sie.edu.cn
}

\begin{abstract}
Combining with the requirements of the development of the smart grid, we raised the research of distribution network monitoring system based on wireless sensor networks. In analysis of the status quo and deficiencies of the existing cable communication system of the distribution network, combining with the structure and characteristics of the wireless sensor networks, we built a new construction strategy of distribution network data monitoring system and discussed it in details regarding both the overall structure itself and the key technologies involved.
\end{abstract}

Keywords: mart grid, wireless sensor network (WSN), power distribution monitoring system, cooperative diversity

\section{Introduction}

Smart grid is capable of benefits of large-scale flexibly access to clean energy, improving the security and stability of the power grid operation, etc., has become a new trend in the network development all over the world. Distribution network connecting the power generation and transmission systems to the power users, it's the last components of power transmission, whose automation degree directly affects the power quality of the user. However, the existing distribution network communication system, due to the limitation of the technical conditions, networking, and other factors, cannot be a good solution to the problems in the actual operation.

Therefore, this paper presents the design of the distribution network monitoring system based on wireless sensor network. Applying the wireless sensor network technology to the power distribution monitoring and management systems, realizing the data acquisition and wireless ad hoc network model based on CC2430 ZigBee chip, adopting cooperative diversity wireless communication technology to improve reliability of the wireless communication in high grid interference environments, and ultimately realizing the dynamic monitoring of the power distribution status data in wireless way, solving the contradiction between the flexibility and robustness of the network system.

\section{The Technical Requirements of the Communication Networking in Distribution Network}

Communication networking is the key technology of power distribution monitoring system, the monitoring system is required of accurately transmitting the control commands from monitoring center to the monitoring terminal and collecting the data of the distribution equipment operation back to the monitoring center. According to the requirements of the distribution network system towards the monitoring object, completing task, data real-time, security and reliability, the technical requirements of power communication network could be divided into the following several aspects: 


\subsection{With the Function of Alarm Timely Communication}

During normal operation, it is able to monitor the running situation of the distribution network. When the system collects operation anomalies, it can automatically send alarm signals and upload to the remote monitoring center, at the same time, the control signals replied by remote monitoring center can also be timely downloaded to the monitoring terminal. The demand of the whole process for the real-time of data transmission is very high [2].

\subsection{With the Function of Regularly Polling or Communication of Uploading Data}

Considering that the distribution monitoring terminal having historical data storage function, the network nodes communicate by using timing or polling mode for saving the communication channel and reducing the loss, so as to avoid the each monitor terminal taking up certain channel communicating with the remote monitoring center for a long time.

\subsection{With the Function of Location Measurement Communication}

Location measurement function is widely applied in the monitoring of the power distribution network. When the monitoring center management needs to look up the operation of some terminal, it is not necessary to request all terminal uploading real-time data, but just to download the control signal to the terminal needed to see. According to the requirement of the instruction, corresponding monitoring terminal can transfer the current real-time data to the remote monitoring center [3].

\subsection{With the Function of Safe and Reliable Data Communication}

Data in a distribution network is roughly divided into two kinds: one is the various parameters and alarm signals that are transmitted from the monitoring terminal to the remote monitoring center; The other is all kinds of control signals being sent by the remote monitoring center. Therefore, the availability and data accuracy of the communication network have a significant influence on the security of power distribution network.

\section{The Structure and Characteristics of Wireless Sensor Networks}

The biggest difference between the wireless sensor networks and the ordinary one is that in addition to the basic communication function, it can also collect data, and process the data basically in the nodes. This characteristic makes the wireless sensor network suitable for the application in the process of monitoring, identifying and controlling. Wireless sensor network nodes with small size, low energy consumption, and dormancy mechanism, can ensure the concealment of the net and running for a long time. In addition, wireless sensor network has good security confidentiality, good scalability and good compatibility to the nodes. As such, its application in the distribution network has a good practical prospect.

\subsection{WSN Network Structure}

WSN system consists of sensor nodes, aggregation nodes and management nodes, the typical structure shown in Figure 1 [1]. In sensor network, nodes are arbitrarily deployed in the monitoring area, forming network by self-organization, transferring monitoring data to the aggregation nodes via multi-hop routing, and eventually with the Internet, wireless network or satellite, transferring data to the management nodes. Users can view, query, search related monitoring data through the management nodes, then configuration and manage the sensor network. 


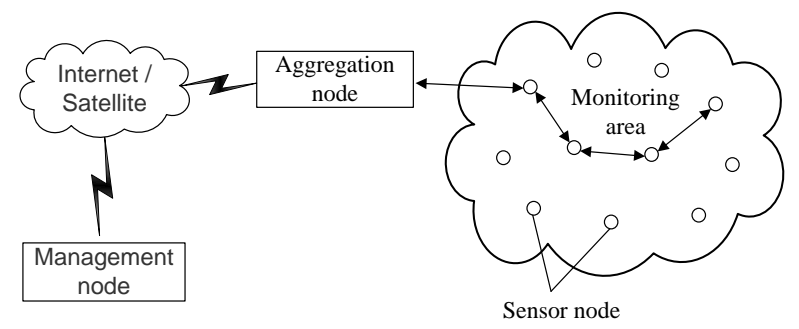

Figure 1. WSN Network Architecture

Being affected by its design, the handling capacity and communication ability of the sensor node cannot be too strong, and it carries limited energy and generally uses batteries. From the network function, each sensor node balances the double functions between the traditional network node terminals and routers. In wireless sensor networks, the basic task of the node is monitoring the information collection to the object and making a preliminary treatment. Moreover it also stores and integrates the data forwarded by the other nodes, cooperating with other nodes to complete some specific task.

The together node is different from other nodes, its ability of communication, processing power and storage is relatively strong, and generally with the external network connection, such as the Internet, to act as a connection point between the wireless sensor network and the external network. At the same time it is also responsible for the task of distributing the monitoring on the management node by the user and forwarding the collected data on the ordinary nodes to the external network. There is not too big difference between the gather nodes and the ordinary sensor nodes, but the node functions have been strengthened, so that in the design of the node, to make it have more memory and computing resources and adequate energy supply, in addition, it can also be the special gateway device without monitoring function only with a wireless communication interface.

\subsection{The Structure of the Sensor Node}

The sensor node in a network is usually a micro embedded system, the hardware structure varies in different applications, but substantially formed by the data acquisition unit, the data processing unit, the data transmission unit and the energy supply unit, as shown in Figure 2 [1].

The data acquisition unit is responsible for monitoring the collection and conversion of the data; Data processing unit in charge of the transceiver of the whole sensor node operating, storage, and the collection of the local data as well as other nodes data; Data transmission unit is responsible for the producing of self-organization network and the transmission of the node data; Power supply unit is responsible for providing the energy for above-mentioned modules' normal operation, the current wireless sensor node generally supplied by the micro batteries.

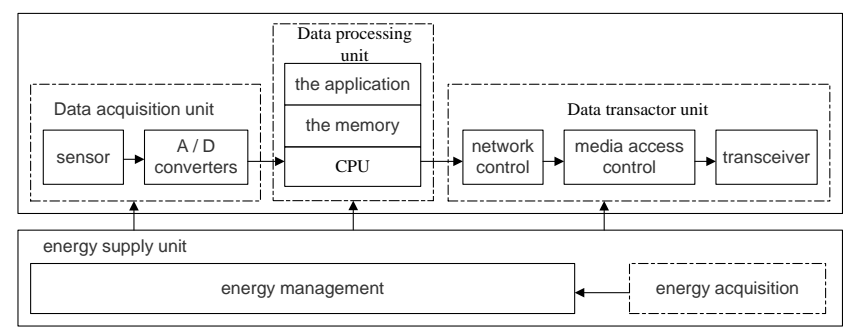

Figure 2. Sensor Node Architecture 


\subsection{WSN Characteristics}

Wireless sensor network compares with the traditional wireless network such as cellular mobile communication network, wireless LAN and Bluetooth networks, although there are similarities, it has its own characteristics [2]. Such as:

Large-scale. Subject to broad monitoring area, in order to avoid the presence of monitoring blind spots, need to deploy a large number of sensor nodes and take advantage of node redundancy to ensure fault tolerance of the system;

Self-organization. In practical applications, WSN generally do not have the network infrastructure, thus require the sensor node with the ability of self-organization;

The limitation of node power energy, the communication ability, computation and storage capacity;

Dynamic. WSN is a dynamic network, all environment interference, node mobility or node failure will result in a topology change, therefore, it requires sensor nodes with the capability of dynamic topology organization;

Multi-hop routing. The WSN network does not have a dedicated routing device, therefore nodes not only have to complete data collection, but also serve the routing task, completing data forwarding of the adjacent nodes;

Applications territorial. Different applications background has different requirements to WSN, its hardware platform, software systems and network protocols vary too. Thus, the difference of network design requirements must be concerned about in practical applications.

\section{The Distribution Network Monitoring System based on WSN}

To introduce wireless sensor networks into the information and communication systems of new generation intelligent distribution network, it should not be regarded as the reconstruction of the existing communication network, but as taking advantage of WSN to complete some condition monitoring towards certain special occasions and special equipments based on the existing network development in order to extend the direct communication between things, to reduce the labor participation in the distribution part and to improve the observability, reliability and economy of distribution network.

\subsection{System Architecture}

Considering the characteristics of the distribution network communications services such as complex wiring, scattered distribution, large amounts of communication points, communications equipment working in poor environment, huge amounts of information etc., the network system architecture can be constructed as shown in Figure 3 [3].

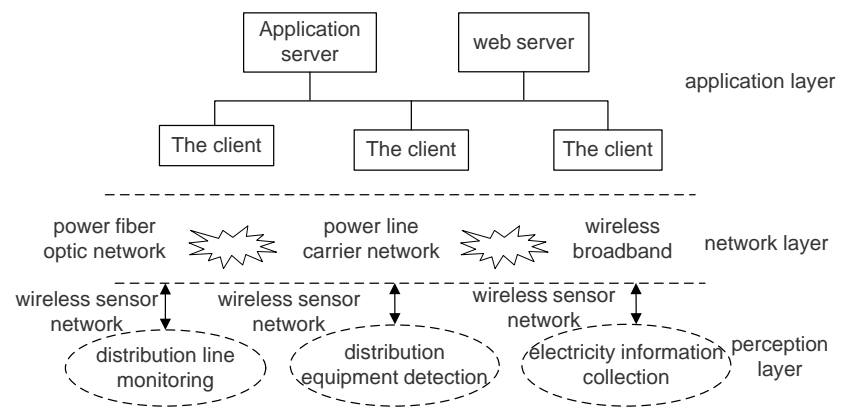

Figure 3. WSN System Structure Applied to the Distribution Network

It is a distributed system composed of the wireless sensor network nodes with the function of temperature and humidity testing, analog and switches detection, gathering nodes and monitoring management center. Measurement and control host, gathering 
nodes and ordinary nodes share the information through the ZigBee technology. The wireless sensor nodes with RF transceiver is distributed in a certain rules in the area needed to be monitored. The central controller at the node completes controlling the sensors within the scope of the monitoring, so as to complete the data collection, preprocessing and transmitting to the gathering node. Measurement and control host realizes the effective control and management of the scene by gathering together the node information. The network node can be set according to different needs to ensure the flexibility and extensibility of the system.

The model is a hierarchical network structure, the perception layer, as the bottom layer, composed of a large number of sensor nodes deployed in the monitoring area, mainly for the acquisition and pooling of the relevant information of the distribution network; The network layer gives priority to power optical fiber communication network, supplemented by the power line carrier communication and wireless broadband network, mainly used to complete the information transmission of various data in perception layer within the wide or local area; The application layer is composed of the web servers and application servers. Application servers, through a smart computing and pattern recognition of received information, achieve a comprehensive analysis and processing of grid information, intelligent decision-making and control of the nodes. Web server is used to provide access to the network services, user information authentication and the establishment of the open interactive environment.

\subsection{Designing of the Data Acquisition Terminal Node}

As the effective supplement of existing main distribution network communication system, the smart sensor technology, signal disposal technology, information fusion technology are applied in the front-end data acquisition and processing of the power distribution automation system, completing accurate monitoring of the power distribution system of some monitoring blind spot. Information such as the temperature, vibration and current leakage of the equipment itself, the humidity information of the equipment's running environment etc. Figure 4 shows the unit design block diagram feeder terminal the model based on CC2430 ZigBee. Input varieties of electric parameters such as voltage, current and harmonic wave into intelligent terminal, or connecting the auxiliary contacts output end of the circuit breaker to the digital quantity input end of the intelligent terminal, can realize the collection of the close-brake and break-brake state and the fault tripping state of the circuit breakers.

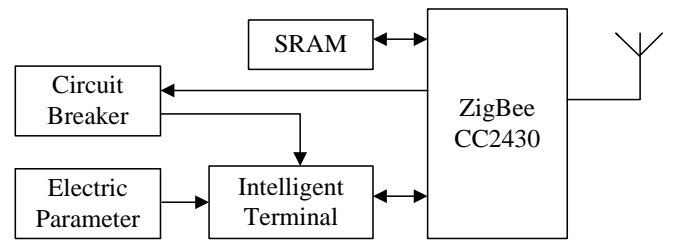

Figure 4. Block Diagram of the Feeder Terminal unit based on ZigBee

According to the characteristics of the distribution network monitoring system, the terminal hardware design is mainly to reduce energy consumption and costs as much as possible under the premise of ensuring nodes application needs. Due to the monitoring of distribution network requiring nodes uninterruptedly working and the energy limit problem of the WSN itself, the node needs not only to have the characteristic of low power consumption, also needs to acquire energy from its environment to achieve power self-supply. Currently available environmental energy collection sources mainly are solar energy, vibration, temperature, airflow and pressure changes etc. 


\subsection{The Analysis of the Key Technology}

\subsubsection{Signal Processing Technology}

In order to ensure the effective operation of the wireless network, sensor nodes must deal with the collected signals promptly and effectively. Considering that the sensor nodes use micro control device with low power consumption, its hardware resources and energy supply are limited, and the more complicated signal processing technology cannot be applied to these nodes, it is necessary to study a signal processing algorithm suitable for ordinary sensor nodes with low power consumption and small capacity.

At present, methods often used can be grouped into time domain analysis and frequency domain analysis. Time domain methods include amplitude and mean variance method, and frequency domain methods include FFT, spectral subtraction and AR spectra [4].

\subsubsection{Communication Protocol Design}

WSN communication protocol includes the physical layer, data link layer, the network layer and the transmission layer. Physical layer is mainly responsible for the production of carrier frequency and signal modulation and demodulation etc., the design of the physical layer is primarily based on selecting the appropriate carrier mode according to the different application background of the distribution network monitoring system; Transmission layer normally use a particular node as a gateway to achieve connections with external network; Data link layer mainly includes the design of media access and error control protocol; While in the network layer, routing protocol is the most critical part, considering different types of data have different time constraints requirement during the data transmission in the system, the designed routing protocol has to realize the validity of energy consumption, and also have a certain degree of self-adaptive ability, so that it could extend the network life cycle and at the same time, meet the time constraints requirement of the applications system and.

\subsubsection{Topology Control and Time Synchronization Mechanism}

As considering the topology control, it is necessary to combine power control with hierarchical topology control and routing protocol design, and to achieve a clustered routing protocol, so as to minimize the nodes energy consumption in the network and effectively extend the life cycle of the network.

In addition, time synchronization is an indispensable mechanism for sensor network systems that need to work together.

The current traditional positioning methods such as network time protocol (NTP), GPS systems, however, due to the considerations of the characteristics of wireless sensor network itself and economy, they are not suitable for application in sensor networks. Thus, how to synchronize between the nodes through the wireless sensor network has become one of the key problems needed to be solved in application of the wireless sensor network. Currently the most widely used three synchronization mechanisms are RBS, TINY/MINI-SYNC and TPSN [5]. RBS mechanism is a kind of synchronization mechanism based on the receiver/receiver clock: a reference group of the node radio clock, the two nodes within the radio range respectively uses the local clock to record the arrival time of reference packet, finally exchange the recording time, realize the clock synchronization between nodes. TINY/MINI-SYNC is a lightweight synchronization mechanism: assume that node's clock drift linear change, then the time migration between the two nodes should also be linear, by exchanging the grouping can estimate the optimal matching offset between two nodes. TPSN uses hierarchy method in the process of time synchronization: according to the hierarchical structure, all the nodes are classified, and with a way of sender/receiver nodes pair, enable every nodes in the network 
synchronization with a node in higher level, so as to realize all the nodes in a wireless sensor network (WSN) having time synchronization with the root node.

\subsubsection{Node Positioning Technology}

Location information is an important part of sensor nodes to collect data, the monitoring data lacking of location information is usually meaningless. In the design of wireless sensor network (WSN), determining the location of the event occurrence or the position of collected data by the nodes is regarded as one of the most basic function of sensor networks. Therefore, after completing the set of the wireless sensor network, the network of sensor nodes must be able to locate themselves, and report it to the electrical personnel on duty when necessary. However, being restricted by design concept of the nodes, the own resources of sensor node is limited, at the same time, The sensor nodes are randomly distributed and its wireless communication distance is limited, while it would only make sense if the data collected by the nodes to combined with its own location within the measurement coordinate system, thus requires the nodes positioning mechanism in the WSN must be able to organize itself, positioning algorithm is a kind of distributed computing, and at the same time, the positioning mechanisms should also have a certain robustness.

According to the location of the node in the network is determined or not, sensor nodes can be divided into the position unknown node and beacon node. The beacon node is the node position that is already known, and as the name implies the location unknown node is unknown, when needing to know the location of these unknown nodes, must determine its position according to the beacon nodes and the positioning mechanism defined in advance. Along with the deepening research on wireless sensor network (WSN), currently there is a lot of positioning mechanism, according to the ways of measuring the nodes' distance or angle in their positioning process, positioning methods in sensor network can be divided into positioning based on distance and positioning regardless of the distance. The current commonly used methods are: trilateration method, maximum likelihood estimation method and triangulation method.

\subsubsection{Data Fusion Technology of Node}

Data fusion is the information processing process that coordinates multiple sources of information from different sensors into a unified feature expression, which is to process information data from multi-sources and to eliminate information defects or redundant contradiction from different information data, and to reduce uncertainty. To effectively utilize the local computing and storage capacity of the nodes, one may adopt the grouping strategy of WSN nodes sensing data based on K-average clustering methods [6], and to process the grouped sensing data with fusion method based on adaptive weighted perception.

The flow of the K-average clustering method processing is as follows: First of all, randomly select k objects, each object represents a cluster of initial average or center. For the rest of the each object, and according to its distance to each cluster center, assign it to the nearest cluster; then recalculate the average of each cluster. Repeat the above process until the criterion function is convergence. Usually adopts the following square error criterion for judgment.

$$
E=\sum_{i=1}^{k} \sum_{p \in C_{i}}\left|p-m_{i}\right|^{2}
$$

In the formula, the "E" is for square error sum of all the objects in the data set, $\mathrm{p}$ is the space point, showing the given data object, $\mathrm{mi}$ is the average of the cluster $\mathrm{Ci}$ ( $\mathrm{p}$ and $\mathrm{m}$ are multidimensional). The principle tried to generate the results of cluster is as compact and independent as possible. 
Data fusion algorithm of wireless sensor network within the cluster based on adaptive weighted: Distributed K-average clustering algorithm based on wireless sensor network (WSN), by processing it, nodes in wireless sensor networks have a more correct data packet. For multiple nodes detection data, however, because of the difference between each sensor measurement accuracy and measurement environment, there must be difference in the accuracy of the measurement. If treating multiple sensors equally, indiscriminately using and processing detection data, must result in the imprecision of the detection results which can lead to the error of the system processing results, sometimes the error is very large. So it is necessary to focus on the position and the detection accuracy of each sensor in the test system, selectively distinguish the importance of the different sensors. This is the basis of adaptive weighted data fusion algorithm.

As for the measurement of accuracy range, in order to weigh the different accuracy of each data, a characteristic figure of "weight" value is quoted to indicate the relative importance of various measured data. The error of high accuracy data is small, so their weight value should be greater; And vice versa, low precision data is large in errors, their weight value should be smaller; To improve the accuracy of the measurement results, all measurements shall be respectively multiplied by their weights value in accordance with the precision, and then take the average. In this paper, the adaptive weighted data fusion method is mainly used in the processing of underlying node detecting data, provides the upper field with more accurate test information and system status information by the time of reducing data redundancy.

Sensor of each node corresponding to different weight value, in the optimal conditions of the minimum mean square error (mse), according to the measured value of the each sensor, with adaptive ways, finding the corresponding optimal weight value, to achieve the optimal in the value of merged $\hat{X}$. Suppose the variance of $n$ sensors are $\sigma_{1}^{2}, \sigma_{2}^{2}$ $\ldots . . \sigma_{n}^{2}$; the true value to be estimated is $\mathrm{X}$, each sensor measurement value are $\mathrm{X} 1, \mathrm{X} 2, \ldots$ $\mathrm{Xn}$, respectively, they are independent to each other and is the unbiased estimation of $\mathrm{X}$; the weighted factors of each sensor are respectively $\mathrm{W} 1, \mathrm{~W} 2, \ldots, \mathrm{Wn}$, then the $\hat{X}$ value after the fusion processing and weighted factor should meet the following two formulas:

$$
\hat{X}=\sum_{p=1}^{n} W_{p} X_{p} \quad \sum_{p=1}^{n} W_{p}=1
$$

The total mean square error is:

$$
\sigma^{2}=E\left[(X-\hat{X})^{2}\right]=E\left[\sum_{p=1}^{n} W_{p}^{2}\left(X-X_{p}\right)^{2}+2 \sum_{\substack{p=1, q=1 \\ p \neq q}}^{n} W_{p} W_{q}\left(X-X_{p}\right)\left(X-X_{q}\right)\right]
$$

The analysis shows that the algorithm has high efficiency and good scalability.

\subsubsection{Cooperative Diversity Issues}

Traditional multi-input/multi-output technology (MIMO) composes of multiple transmitting/receiving antenna diversity through configuring multiple antennas at the transmitting/ receiving end. However, for small wireless terminals, this is clearly unrealistic. Therefore it leads to the difficulty in the practical use of the MIMO technology applied in wireless sensor network [7]. Cooperative diversity found a way in utilizing MIMO technology to small wireless terminal, taking the various advantages of MIMO technology, can effectively use the space resources to improve the performance of communication system.

The anti-jamming mechanism of the cooperative diversity compares with the basic method of anti-interference (e.g., direct sequence spread spectrum, frequency hopping, jumping), the traditional methods have no use of space resources. The working principle of frequency hopping is the signal carrier frequency controlled and jumped by pseudo random code or a specific frequency hopping pattern. The size of the frequency hopping gain depends on the amplitude and the speed of the frequency hopping, and jumping 
technique in time have similar conclusions. Cooperative diversity technology from another angle can be thought as according to certain criteria (a particular empty jump pattern), the user information jumping on the spatial orientation, its empty jumping gain can compare with time hopping and frequency hopping gain. As the increase of users, empty jumping range and empty jumping speed, the gain will be greater.

Set the wireless sensor network link as ratio of the instantaneous signal noise, $\mathrm{C}(\gamma)$ as link capacity, $\mathrm{P}(\gamma)$ as the probability distribution density of SNR, R as the rate at which the unit bandwidth required, when the link satisfies $R>C(\gamma)=l b(1+\gamma)$, namely transfer rate over channel capacity will generate interrupt event, the probability distribution depends on the link of average signal-to-noise ratio and its channel fading distribution model. Actual channel gain usually satisfy Rayleigh fast fading probability distribution, namely the instantaneous channel SNR meet the index distribution of parameters for $1 / \Gamma$, $\Gamma$ is the SNR after fast decline. At this point the interrupt probability of Pout can be expressed as [8].

$$
P_{\text {out }}=\int_{0}^{2 R-1} p(\gamma) d \gamma=\int_{0}^{2 R-1} \frac{1}{\Gamma} \exp \left(-\frac{\gamma}{\Gamma}\right) d \gamma=1-\exp \left(-\frac{2^{R}-1}{\Gamma}\right)
$$

The result of the above applies only to the interrupt probability calculation of a single link, in practice, often appear the interrupt structure of multiple paths. Assuming that there is no visibility link between the source and the destination, what the destination receives are the signals sent by multiple relay nodes. Under such circumstances, the interrupt probability of this case will be multiple probability integral calculation, the integral can be expressed as follows:

$$
P_{\text {out }}=\int_{F} \ldots \prod_{j \in S_{i}} p\left(\gamma_{i}\right) d \gamma_{i}
$$

In the formula, $\mathrm{Si}$ is probability set with relay being selected. $\mathrm{F}$ can be represented as:

$$
F=\left\{1+\sum_{j \in S_{i}} \gamma_{i}<2^{\alpha R}\right\}
$$

In the formula, ${ }^{\alpha=|S|+1}$ is coordination relay set of Si's the ith source node. following the increase of relay, the effective integral areas of the interrupt probability increase. Therefore, in order to minimize the interrupt probability, should choose a set of coordination relay set.

$$
S_{i}=\arg \min _{D_{i}^{(S)}} P_{\text {out }, i}\left(S_{i}\right)
$$

Obviously, Si can only be selected from users' collection Di(s) that are able to receive the ith users broadcast data, namely $S_{i} \in D_{i}^{(s)}$.

The relay selection strategy is selecting one of the largest SNR node in candidate set [7], which will only select maximum SNR relay nodes as a cooperative relay. In this way, the relay limit to 1 , so that the network structure is simple, greatly reduce computational complexity, is an ideal relay selection strategy.

\section{Conclusion}

Wireless sensor network is a novel technology about acquiring and processing information, and the biggest difference between WSN and the traditional distribution network communication system is that in addition to the basic communication function, it can also collect data and perform basic processing of these data in the node. This feature makes the wireless sensor network quite adaptive to those links such as the data acquisition and condition monitoring of distribution network. With those characteristics of low power consumption, low-cost, distributed and self-organization, it shows the incomparable advantages to the traditional technology and can effectively supplement the existing distribution network communication mode, which has a good application prospect in the distribution network. 
The case proposed in this paper, that the distribution network monitoring based on wireless sensor networks, discuss more about how to conduct data collection quickly and accurately, and not enough about the research of network reconstruction, recovery after power failure and Peer-to-peer network topology network. in addition, since the ZigBee works in the free range of $2.4 \mathrm{gHz}$, which is applied by lots of wireless communication technologies, the network is largely interfered by others. in next step, this issue should be conducted in-depth research.

\title{
Acknowledgement
}

This work was supported by This work is supported by National Nature Science Foundation of China under Grant 61304069, 61372195, 61371200, the Fundamental Research Fund of Liaoning Provincial Education Department Key Laboratory under Grant LZ2014050 and the Scientific Research Fund of Liaoning Provincial Education Department under Grant L2012468, L2013494). The authors also gratefully acknowledge the helpful comments and suggestions of the reviewers, which have improved the presentation.

\section{References}

[1] X. Yang, "Wireless Sensor Networks for Real-Time Monitoring”, People's Posts and Telecommunications Press, (2010).

[2] Y. Q. Fan and J. Mi, "Application of Wireless Private Network Technology in Smart Distribution Network", Computer Knowledge and Technology, vol. 1103, (2011).

[3] J. Liu, "First Exploration of Things in Oilfield Distribution Grid", Instrument Electrical, vol. 60, (2011).

[4] X. H. Hu and Y. Zhao, "Study of Impacts of Two Types' Distributed Generation on Distribution Network Voltage Sags", Power System Technology and IEEE Power India Conference, vol. 102, (2008).

[5] Y. G. Wang and X. G. Yin, "Wireless Sensor Networks in Substation Automation System Technology", Grid Technology, vol. 20, (2009).

[6] L. Y. Zhou, "Application of New Generation Communication Technology in Automation of Power Distribution Grid", East China Electric Power, (2009).

[7] X. H. He, "Relay Technology in the Next Generation Mobile Communication System", Journal of Mobile Communications, vol. 40, (2008).

[8] Y. J. Tang, "Power Distribution Monitoring System based on Wireless Sensor Network Design", Journal of Low-Voltage Electrical Appliances, (2011).

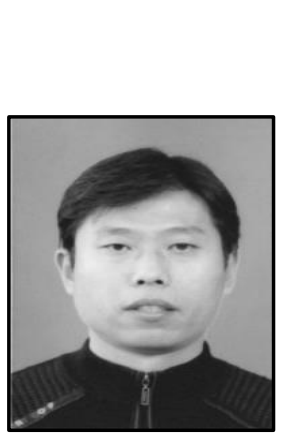

\begin{abstract}
Authors
Shihai Ma, He was born in 1977, at Chaoyang, Liaoning. He is the Senior engineer, and the main research direction is power system automation and network communication technology.
\end{abstract}

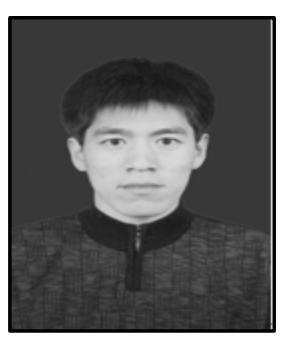

Yan Zhao, He received the Ph.D. degree in control engineering from Northeastern University, Shenyang, China, in 2008.

$\mathrm{He}$ is currently an associate professor with the School of Renewable Energy, Shenyang Institute of Engineering, Shenyang. His current research interests include fuzzy control theory and distributed generation technique. 


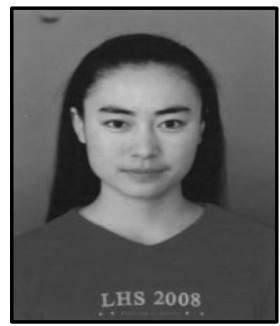

Haiyang Zhang, She was born in 1979, at Chaoyang, Liaoning. She is a lecturer. Her main research direction is power system automation and network communication technology. 
International Journal of Hybrid Information Technology Vol.9, No.3 (2016) 